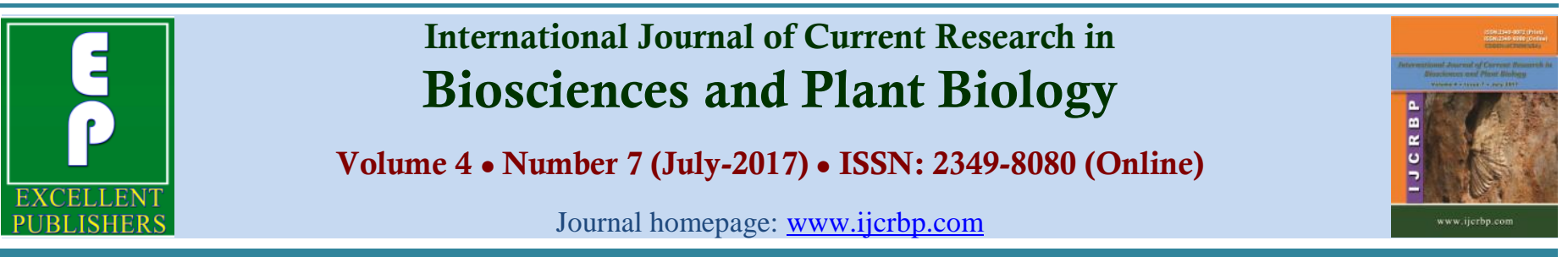

\title{
Synthesis and Characterization Platinum (II), Palladium (II) and Nickel (II) Complexes with some Heterocyclic Ligand of 1,3,4-Oxadazole Derivative
}

\author{
Hikmat Ali Mohamad ${ }^{1 *}$, Widad Taha Al-Kattan² and Ali Nafia Najem² \\ ${ }^{I}$ College of Education, University of Salahaddin-Erbil, Iraq \\ ${ }^{2}$ College of Science, Mosul University-Mosul, Iraq \\ *Corresponding author.
}

\begin{abstract}
The complexes of $\mathrm{Ni}(\mathrm{II}), \mathrm{Pd}(\mathrm{II})$ and $\mathrm{Pt}(\mathrm{II})$ with four 1,3,4-oxadiazole derivatives ligand have been synthesized and characterized which are obtained by the reaction of metal chloride salt with 2,5-diphenyl-1,3,4-oxadiazol (PPD), 2-(4-biphenylyl)-5-phenyl-1,3,4oxadiazol (PBD), 2-amino-5-phenyl-1,3,4-oxadiazol (APOD) and 2,5-Bis(4diethylaminophenyl)-1,3,4-oxadiazole (DAPO), in (1:2) metal : ligand molar ratio. The complexes have been characterized using molar conductivity, magnetic susceptibility, microanalysis elemental analysis (C.H.N), atomic absorption, spectroscopic (IR, UV-Vis and ${ }^{1} \mathrm{H}-\mathrm{NMR},{ }^{13} \mathrm{C}-\mathrm{NMR}$ ).
\end{abstract}

\section{Article Info}

Accepted: 30 June 2017

Available Online: 06 July 2017

\section{Keywords}

Biologically active complexes

Heterocyclic ligand

$\mathrm{Ni}(\mathrm{II}), \mathrm{Pd}(\mathrm{II}), \mathrm{Pt}(\mathrm{II})$ complexes

\section{Introduction}

Oxadiazole, heterocyclic nucleus has a wide attention of the chemist in search for the new therapeutic molecules (Somani and Shirodkar, 2009). Among them, 1,3,4oxadiazole is considered to be derived from furan by replacement of two methane $(-\mathrm{CH}=)$ groups, by two pyridine type nitrogen $(-\mathrm{N}=)$. 1,3,4-oxadiazole is a cyclic compound containing one oxygen and two nitrogen atoms in five membered ring (Bala et al., 2010). It is known that the ligand forms complexes with almost every metal ion of the d-block transition elements (Al-obaidi et al., 2004), have exhibited a wide range of biological properties including anti-bacterial, anti-viral, anti-fungal, anti-cancer, anti-tumor, antiinflammatory, anti-hypertensive, anti-convulsant and anti-diabetic properties (Kavitha et al., 2014). 1,3,4Oxadiazole derivatives are also among the most widely employed electron conducting and hole blocking (ECHB) materials in organic light-emitting diodes (LEDs) (Gudasi et al., 2007). We were presented here the preparation and characterization of $\mathrm{Ni}, \mathrm{Pd}$ and $\mathrm{Pt}$ complexes.

\section{Materials and methods}

\section{Experimental}

All chemicals were of reagent grade, were used as supplied TCI, Canspec, Sigma, Solarbio, Fluka, Scharlue and $\mathrm{CDH}$. Various analysis carried out were: Conductivity measurements for $10^{-3} \mathrm{M}$ solution of the complexes in (DMSO), elemental analysis (C.H.N)Infrared spectra, the UV/Vis in DMSO solvent, melting point, magnetic susceptibility, ${ }^{1} \mathrm{H}-\mathrm{NMR}$ and ${ }^{13} \mathrm{C}-\mathrm{NMR}$ spectra of complexes in DMSO as a solvent. 
Synthesis of $\left[\mathrm{Ni}(\mathrm{PPD})_{2}\left(\mathrm{H}_{2} \mathrm{O}\right)_{2} \mathrm{Cl}_{2}\right] .4 \mathrm{H}_{2} \mathrm{O}$

A solution of (PPD) (1mmol, $0.22 \mathrm{~g})$ in methanol $(10 \mathrm{ml})$ was added to a solution of $(0.5 \mathrm{mmol}, 0.12 \mathrm{~g}) \mathrm{NiCl}_{2} \cdot 6 \mathrm{H}_{2} \mathrm{O}$ in methanol $(10 \mathrm{ml})$, the mixture was refluxed for $(15$ hrs) with stirring. The mixture was filtered off, washed with methanol $(10 \mathrm{ml})$, and then dried under vacuum for several hours.

\section{Synthesis of $\left[\mathrm{Pt}(\mathrm{PPD})_{2} \mathrm{Cl}_{2}\right]$}

A solution of (PPD) (1mmol, $0.22 \mathrm{~g})$ in methanol $(10 \mathrm{ml})$ was added to a solution of $(0.5 \mathrm{mmol}, 0.21) \mathrm{K}_{2} \mathrm{PtCl}_{4}$ in methanol $(10 \mathrm{ml})$. The mixture was refluxed for $(25 \mathrm{~h})$ with stirring. The precipitate was filtered off, washed with methanol $(10 \mathrm{ml})$, diethylether $(10 \mathrm{ml})$ and dried in oven $\left(50-55^{\circ} \mathrm{C}\right)$ for several hours.

\section{Synthesis of $\left[\mathrm{Ni}(\mathrm{PBD})_{2}\left(\mathrm{H}_{2} \mathrm{O}\right)_{2} \mathrm{Cl}_{2}\right] . \mathrm{H}_{2} \mathrm{O}$}

A solution of (PBD) (1mmol, 0.3g) in dichloromethane (10ml) was added to a solution of $(0.5 \mathrm{mmol}, 0.12 \mathrm{~g})$ $\mathrm{NiCl}_{2} \cdot 6 \mathrm{H}_{2} \mathrm{O}$ in methanol $(10 \mathrm{ml})$. The mixture was refluxed for (15 hrs) with stirring. The Mixture was filtered off washed with methanol $(10 \mathrm{ml})$, and then dried under vacuum for several hours.

\section{Synthesis of $\left[\mathrm{M}(\mathrm{PBD})_{2} \mathrm{Cl}_{2}\right](\mathrm{M}=\mathrm{Pd}, \mathrm{Pt})$}

A solution of (PBD) (1mmol, 0.3g) in dichloromethane $(10 \mathrm{ml})$ was added to a solution of $(0.5 \mathrm{mmol}) \mathrm{PdCl}_{2}$ $(0.09 \mathrm{~g})$ and $\mathrm{K}_{2} \mathrm{PtCl}_{4}(0.21)$ in methanol $(10 \mathrm{ml})$. The mixture was refluxed for (3-20 hrs) with stirring. The precipitate was filtered off, washed with methanol $(10 \mathrm{ml})$, dichloromethane $(10 \mathrm{ml})$ and dried in oven $(50-$ $55^{\circ} \mathrm{C}$ ) for several hours.

\section{Synthesis of $\left[\mathrm{M}(\mathrm{APOD})_{2} \mathrm{Cl}_{2}\right](\mathrm{M}=\mathrm{Ni}, \mathrm{Pd}, \mathrm{Pt})$}

A solution of (APOD) (1mmol, 0.16g) in methanol $(10 \mathrm{ml})$ was added to a solution of $(0.5 \mathrm{mmol})$ $\mathrm{NiCl}_{2} \cdot 6 \mathrm{H}_{2} \mathrm{O}(0.12 \mathrm{~g}), \mathrm{PdCl}_{2}(0.09 \mathrm{~g})$ and $\mathrm{K}_{2} \mathrm{PtCl}_{4}(0.21)$ in methanol $(10 \mathrm{ml})$. The mixture was refluxed for (3-15 hrs) with stirring. The precipitate was filtered off, washed with methanol $(10 \mathrm{ml})$, dried in oven $\left(50-55^{\circ} \mathrm{C}\right)$ for several hours.

\section{Synthesis of $\left[\mathrm{Ni}(\mathrm{DAPO})_{2} \mathrm{Cl}_{2}\right] \cdot 6 \mathrm{H}_{2} \mathrm{O}$}

A solution of (APOD) (1mmol, $0.36 \mathrm{~g}$ ) in methanol $(10 \mathrm{ml})$ was added to a solution of $(0.5 \mathrm{mmol})$
$\mathrm{NiCl}_{2} \cdot 6 \mathrm{H}_{2} \mathrm{O}(0.12 \mathrm{~g}), \mathrm{PdCl}_{2}(0.09 \mathrm{~g})$ and $\mathrm{K}_{2} \mathrm{PtCl}_{4}(0.21)$ in methanol $(10 \mathrm{ml})$. The mixture was refluxed for $(12 \mathrm{hrs})$ with stirring. The precipitate was filtered off, washed with methanol $(10 \mathrm{ml})$, dried in oven $\left(50-55^{\circ} \mathrm{C}\right)$ for several hours.

\section{Synthesis of $\left[\mathrm{M}(\mathrm{DAPO})_{2} \mathrm{Cl}_{2}\right](\mathrm{M}=\mathrm{Pd}, \mathrm{Pt})$}

A solution of (APOD) (1mmol, 0.36g) in methanol $(10 \mathrm{ml})$ was added to a solution of $(0.5 \mathrm{mmol}), \mathrm{PdCl}_{2}$ $(0.09 \mathrm{~g})$ and $\mathrm{K}_{2} \mathrm{PtCl}_{4}(0.21)$ in methanol $(10 \mathrm{ml})$. The mixture was refluxed for (3-27 hrs) with stirring. The precipitate was filtered off, washed with methanol $(10 \mathrm{ml})$, dried in oven $\left(50-55^{\circ} \mathrm{C}\right)$ for several hours.

\section{Results and discussion}

The complexes were prepared through direct reaction of $\mathrm{Ni}(\mathrm{II}), \mathrm{Pd}(\mathrm{II})$ and $\mathrm{Pt}(\mathrm{II})$ chloride salt with ligands in a (2:1)molar ratio that gave a neutral complexes. All the complexes are colored and soluble in DMSO. The molar conductivity in $10^{-3} \mathrm{M}$ (DMSO) solution are in the (1020) $\mathrm{ohm}^{-1} \mathrm{~cm}^{2} \mathrm{~mol}^{-1}$ range indicating a non-electrolytic nature complexes. This is in consistent with the stoichiometry assumed for the complexes on the basis of the analytical data. The physical analytical data of complexes given in Table 1, were in agreement with the calculated values. The suggested molecular formulas were also supported by subsequent spectral and magnetic moment measurement. The proposed structure of metal complexes is given in Fig. 1. Various spectral analyses of metal(II) complexes are depicted in Fig. 2.

Magnetic susceptibility measurements: The magnetic moments were measured at $25^{\circ} \mathrm{C}$. The results indicated octahedral for complexes (1-3) and square planner for complexes (4-11) (Cotton and Wikinson, 1999; Blake et al., 1993).

Electronic spectral studies: The electronic spectra of the ligands and their complexes in $10^{-3} \mathrm{M}$ solution DMSO was recorded, Table 2 exhibits the bands at 218$225 \mathrm{~nm}$ and $265-308 \mathrm{~nm}$ were due to $\pi-\pi^{*}$ and $n-\pi^{*}$ transition within the ligands. The UV-visible spectra of complex(1-3) have been given two spins allowed transitions at (14409-14430) $\mathrm{cm}^{-1}\left(\mathrm{v}_{2}\right)$ and (22831$22988) \mathrm{cm}^{-1}\left(\mathrm{~V}_{3}\right)$, were assigned to transitions ${ }^{3} \mathrm{~A}_{2} \mathrm{~g} \rightarrow{ }^{3} \mathrm{~T}_{1} \mathrm{~g}(\mathrm{~F})$ and ${ }^{3} \mathrm{~A}_{2} \mathrm{~g} \rightarrow{ }^{3} \mathrm{~T}_{1} \mathrm{~g}(\mathrm{P})$ respectively, it's reasonable to assigned octahedral geometry (Levason et al., 1976). The UV-visible spectrum of complexes (411) have been given spins allowed transitions at (21459- 
$21739) \mathrm{cm}^{-1}\left(\mathrm{~V}_{1}\right),(24875-25000) \mathrm{cm}^{-1}\left(\mathrm{~V}_{2}\right)$, and $(29850-$ $32573) \mathrm{cm}^{-1}\left(v_{3}\right)$ were assigned to transitions ${ }^{1} \mathrm{~A}_{1} \mathrm{~g} \rightarrow{ }^{1} \mathrm{~A}_{2} \mathrm{~g}$, ${ }^{1} \mathrm{~A}_{1} \mathrm{~g} \rightarrow{ }^{1} \mathrm{~B}_{1} \mathrm{~g}$ and ${ }^{1} \mathrm{~A}_{1} \mathrm{~g} \rightarrow{ }^{1} \mathrm{Eg}$ respectively as it show in
Table 2, these transitions value are indicated to square planner geometry (Park and Cho, 1989; Qadir et al., 2004).

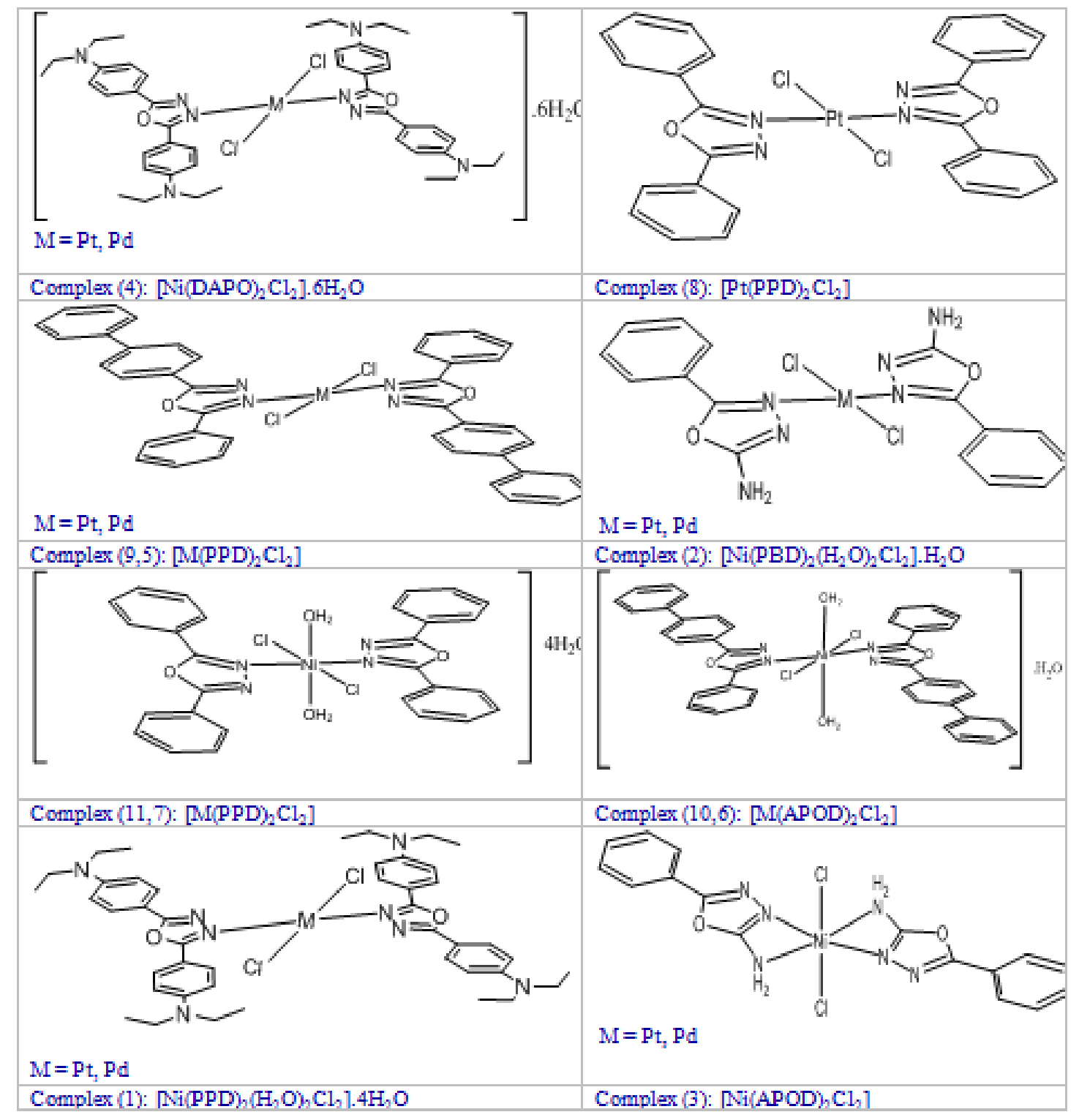

Fig. 1: Proposed structures for the metal(II) complexes.

Infrared spectral studies: The IR spectra of the free ligand and those of the prepared complexes are tabulated in Table 3. The infrared spectrum of APOD showed bands in the range (3396-3269) $\mathrm{cm}^{-1}$, which are corresponding to $v(\mathrm{NH})$ of amine group (Galal et al., 2010; Tomi, 2012). A shifted with change in shape were observed from these bands, while increasing in intensity were noticed. The significant may be a result of coordination with metal ion. A strong absorption at
$1610,1613,1618$ and $1653 \mathrm{~cm}^{-1}$ appeared in a spectrum of PPD, PBD, APOD and DAPO respectively which indicated to the $v(\mathrm{C}=\mathrm{N})$ of oxadiazole ring, a negative shift in $v(\mathrm{C}=\mathrm{N})$ indicates the involvement of nitrogen in coordination (Subha Selvaraj, 2014; Patricia et al., 2002). They also showed a band in the region 416$525 \mathrm{~cm}^{-1}$ which may be due to $v(\mathrm{M}-\mathrm{N})$. A broad band in the range (3410-3357) $\mathrm{cm}^{-1}$ can be assigned to $v(\mathrm{OH})$ and the complexes $(1,2)$ were exhibited bands in the 
region $685-922 \mathrm{~cm}^{-1}$ which may be assignable to the coordinated water, which is absent in the free ligand (Aggarwal and Singh, 1975).

${ }^{1}$ HNMRdata: ${ }^{1}$ HNMR of the ligand was recorded using $\mathrm{d}_{6}(\mathrm{DMSO})$ as a solvent. The spectrum of the APOD showed single signal at $(\delta=7.65)$ due to $\mathrm{N}-\mathrm{H}$ proton that shifted to high field at $(\delta=3.3)$ for complex
(3) due to linkage with metal ion (Table 4). The aromatic ring proton show a multi signal at $(\delta=7.65$, 8.98) for PPD, $(\delta=7.38,7.75,8.08)$ for PBD, $(\delta=7.6$, 7.8) for APOD, $(\delta=6.8,7,7)$ in a free ligand, the spectra show none change in this signal value for the prepared complexes which indicate non-participation carbon atom of the aromatic ring in the coordination (Garces et al., 1988).

Table 1. The physical analytical data of complexes.

\begin{tabular}{|c|c|c|c|c|c|c|c|c|c|}
\hline & \multirow{2}{*}{ Complexes } & \multirow{2}{*}{ Meff } & \multirow{2}{*}{ Color } & \multirow{2}{*}{ Melting point } & \multirow{2}{*}{$\begin{array}{l}\text { Product } \\
\%\end{array}$} & \multirow[t]{2}{*}{ М\% } & \multicolumn{3}{|l|}{ C.H.N } \\
\hline & & & & & & & $\mathrm{C} \%$ & H\% & N\% \\
\hline 1 & {$\left[\mathrm{Ni}(\mathrm{PPD})_{2}\left(\mathrm{H}_{2} \mathrm{O}\right)_{2} \mathrm{Cl}_{2}\right] \cdot 4 \mathrm{H}_{2} \mathrm{O}$} & 2.92 & Pale green & $225-228$ & 79 & $\begin{array}{l}8.38 \\
(8.60)\end{array}$ & $\begin{array}{l}49.682 \\
(49.30)\end{array}$ & $\begin{array}{l}4.681 \\
(4.73)\end{array}$ & $\begin{array}{l}7.989 \\
(8.21)\end{array}$ \\
\hline 2 & {$\left[\mathrm{Ni}(\mathrm{PBD})_{2}\left(\mathrm{H}_{2} \mathrm{O}\right)_{2} \mathrm{Cl}_{2}\right] \cdot \mathrm{H}_{2} \mathrm{O}$} & 2.861 & Pale green & $195-197$ & 77 & $\begin{array}{l}7.85 \\
(7.50)\end{array}$ & $\begin{array}{l}61.85 \\
(61.57)\end{array}$ & $\begin{array}{l}4.66 \\
(4.39)\end{array}$ & $\begin{array}{l}6.804 \\
(7.16)\end{array}$ \\
\hline 3 & {$\left[\mathrm{Ni}(\mathrm{APOD})_{2} \mathrm{Cl}_{2}\right]$} & 3.099 & Green & $270-273$ & 93 & $\begin{array}{l}11.45 \\
(12.05)\end{array}$ & $\begin{array}{l}43.194 \\
(42.52)\end{array}$ & $\begin{array}{l}3.207 \\
(3.12)\end{array}$ & $\begin{array}{l}18.669 \\
(18.60)\end{array}$ \\
\hline 4 & {$\left[\mathrm{Ni}(\mathrm{DAPO})_{2} \mathrm{Cl}_{2}\right] \cdot 6 \mathrm{H}_{2} \mathrm{O}$} & Dia & $\begin{array}{l}\text { Dark } \\
\text { green }\end{array}$ & $257-254$ & 77 & $\begin{array}{l}6.12 \\
(6.84)\end{array}$ & $\begin{array}{l}55.396 \\
(54.67)\end{array}$ & $\begin{array}{l}7.292 \\
(7.09)\end{array}$ & $\begin{array}{l}11.196 \\
(11.59)\end{array}$ \\
\hline 5 & {$\left[\mathrm{Pd}(\mathrm{PBD})_{2} \mathrm{Cl}_{2}\right]$} & Dia & $\begin{array}{l}\text { Pale } \\
\text { yellow }\end{array}$ & No melting up 300 & 94 & $\begin{array}{l}13.01 \\
(13.75)\end{array}$ & $\begin{array}{l}62.042 \\
(62.01)\end{array}$ & $\begin{array}{l}3.653 \\
(3.65)\end{array}$ & $\begin{array}{l}6.929 \\
(7.24)\end{array}$ \\
\hline 6 & {$\left[\mathrm{Pd}(\mathrm{APOD})_{2} \mathrm{Cl}_{2}\right]$} & Dia & Pale brown & No melting up 300 & 80 & $\begin{array}{l}20.02 \\
(21.30)\end{array}$ & $\begin{array}{l}37.905 \\
(38.31)\end{array}$ & $\begin{array}{l}2.903 \\
(3.21)\end{array}$ & $\begin{array}{l}16.96 \\
(16.75)\end{array}$ \\
\hline 7 & {$\left[\mathrm{Pd}(\mathrm{DAPO})_{2} \mathrm{Cl}_{2}\right]$} & Dia & $\begin{array}{l}\text { Dark } \\
\text { yellow }\end{array}$ & $242 \mathrm{~d}$ & 69 & $\begin{array}{l}11.84 \\
(11.74)\end{array}$ & $\begin{array}{l}57.714 \\
(58.31)\end{array}$ & $\begin{array}{l}6.02 \\
(6.23)\end{array}$ & $\begin{array}{l}12.251 \\
(12.36)\end{array}$ \\
\hline 8 & {$\left[\mathrm{Pt}(\mathrm{PPD})_{2} \mathrm{Cl}_{2}\right]$} & Dia & Pale brown & No melting up 300 & 66 & $\begin{array}{l}26.87 \\
(27.46)\end{array}$ & $\begin{array}{l}47.9 \\
(47.3)\end{array}$ & $\begin{array}{l}3.03 \\
(2.84)\end{array}$ & $\begin{array}{l}8.01 \\
(7.89)\end{array}$ \\
\hline 9 & {$\left[\mathrm{Pt}(\mathrm{PBD})_{2} \mathrm{Cl}_{2}\right]$} & Dia & Brown & $243 d$ & 70 & $\begin{array}{l}22.23 \\
(22.61)\end{array}$ & $\begin{array}{l}54.21 \\
(55.56)\end{array}$ & $\begin{array}{l}3.36 \\
(3.27)\end{array}$ & $\begin{array}{l}6.52 \\
(6.48)\end{array}$ \\
\hline 10 & {$\left[\mathrm{Pt}(\mathrm{APOD})_{2} \mathrm{Cl}_{2}\right]$} & Dia & Brown & No melting up 300 & 71 & $\begin{array}{l}31.95 \\
(33.16)\end{array}$ & $\begin{array}{l}31.5 \\
(32.67)\end{array}$ & $\begin{array}{l}2.1 \\
(2.40)\end{array}$ & $\begin{array}{l}14.44 \\
(14.29)\end{array}$ \\
\hline 11 & {$\left[\mathrm{Pt}(\mathrm{DAPO})_{2} \mathrm{Cl}_{2}\right]$} & Dia & Yellow & $259 \mathrm{~d}$ & 69 & $\begin{array}{l}18.69 \\
(19.61)\end{array}$ & $\begin{array}{l}52.72 \\
(53.12)\end{array}$ & $\begin{array}{l}5.45 \\
(5.67)\end{array}$ & $\begin{array}{l}10.98 \\
(11.24)\end{array}$ \\
\hline
\end{tabular}

Table 2. UV-Vis spectra of prepared complexes.

\begin{tabular}{|c|c|c|c|c|c|}
\hline & \multirow{2}{*}{ Complexes } & \multicolumn{2}{|c|}{ Absorption band } & \multirow[b]{2}{*}{ Transitions } & \\
\hline & & $\mathbf{c m}^{-1}$ & $\mathrm{~nm}$ & & \\
\hline 1 & {$\left[\mathrm{Ni}(\mathrm{PPD})_{2}\left(\mathrm{H}_{2} \mathrm{O}\right)_{2} \mathrm{Cl}_{2}\right] \cdot 4 \mathrm{H}_{2} \mathrm{O}$} & $\begin{array}{l}14409 \\
22831 \\
34965\end{array}$ & $\begin{array}{l}694 \\
438 \\
286\end{array}$ & $\begin{array}{l}{ }^{3} \mathrm{~A}_{2} \mathrm{~g} \rightarrow{ }^{3} \mathrm{~T}_{1} \mathrm{~g}(\mathrm{~F}) \\
{ }^{3} \mathrm{~A}_{2} \mathrm{~g} \rightarrow{ }^{3} \mathrm{~T}_{1} \mathrm{~g}(\mathrm{P}) \\
\text { C. } \mathrm{T}\end{array}$ & $\mathrm{O}_{\mathrm{h}}$ \\
\hline 2 & {$\left[\mathrm{Ni}(\mathrm{PBD})_{2}\left(\mathrm{H}_{2} \mathrm{O}\right)_{2} \mathrm{Cl}_{2}\right] . \mathrm{H}_{2} \mathrm{O}$} & $\begin{array}{l}14430 \\
22988 \\
32679\end{array}$ & $\begin{array}{l}781 \\
435 \\
306\end{array}$ & $\begin{array}{l}{ }^{3} \mathrm{~A}_{2} \mathrm{~g} \rightarrow{ }^{3} \mathrm{~T}_{1} \mathrm{~g}(\mathrm{~F}) \\
{ }^{3} \mathrm{~A}_{2} \mathrm{~g} \rightarrow{ }^{3} \mathrm{~T}_{1} \mathrm{~g}(\mathrm{P}) \\
\mathrm{C} . \mathrm{T}\end{array}$ & $\mathrm{O}_{\mathrm{h}}$ \\
\hline 3 & {$\left[\mathrm{Ni}(\mathrm{APOD})_{2} \mathrm{Cl}_{2}\right]$} & $\begin{array}{l}14430 \\
22935 \\
34722\end{array}$ & $\begin{array}{l}788 \\
693 \\
436\end{array}$ & $\begin{array}{l}{ }^{3} \mathrm{~A}_{2} \mathrm{~g} \rightarrow{ }^{3} \mathrm{~T}_{1} \mathrm{~g}(\mathrm{~F}) \\
{ }^{3} \mathrm{~A}_{2} \mathrm{~g} \rightarrow{ }^{3} \mathrm{~T}_{1} \mathrm{~g}(\mathrm{P}) \\
\text { C. } \mathrm{T}\end{array}$ & $\mathrm{O}_{\mathrm{h}}$ \\
\hline 4 & {$\left[\mathrm{Ni}(\mathrm{DAPO})_{2} \mathrm{Cl}_{2}\right] \cdot 6 \mathrm{H}_{2} \mathrm{O}$} & $\begin{array}{l}23809 \\
27472 \\
32154\end{array}$ & $\begin{array}{l}420 \\
364 \\
311\end{array}$ & $\begin{array}{l}{ }^{1} \mathrm{~A}_{1} \mathrm{~g} \rightarrow{ }^{1} \mathrm{~B}_{1} \mathrm{~g} \\
{ }^{1} \mathrm{~A}_{1} \mathrm{~g} \rightarrow{ }^{1} \mathrm{Eg} \\
\text { C.T }\end{array}$ & $\mathrm{Sp}$ \\
\hline 5 & {$\left[\mathrm{Pd}(\mathrm{PBD})_{2} \mathrm{Cl}_{2}\right]$} & $\begin{array}{l}24937 \\
32679\end{array}$ & $\begin{array}{l}401 \\
306\end{array}$ & $\begin{array}{l}{ }^{1} \mathrm{~A}_{1} \mathrm{~g} \rightarrow{ }^{1} \mathrm{~B}_{1} \mathrm{~g} \\
{ }^{1} \mathrm{~A}_{1} \mathrm{~g} \rightarrow{ }^{1} \mathrm{Eg}\end{array}$ & $\mathrm{Sp}$ \\
\hline 6 & {$\left[\mathrm{Pd}(\mathrm{APOD})_{2} \mathrm{Cl}_{2}\right]$} & $\begin{array}{l}24937 \\
34722\end{array}$ & $\begin{array}{l}401 \\
288\end{array}$ & $\begin{array}{l}{ }^{1} \mathrm{~A}_{1} \mathrm{~g} \rightarrow{ }^{1} \mathrm{~B}_{1} \mathrm{~g} \\
\text { C. } \mathrm{T}\end{array}$ & $\mathrm{Sp}$ \\
\hline
\end{tabular}




\begin{tabular}{|c|c|c|c|c|c|}
\hline 7 & {$\left[\mathrm{Pd}(\mathrm{DAPO})_{2} \mathrm{Cl}_{2}\right]$} & $\begin{array}{l}25000 \\
27624 \\
32154\end{array}$ & $\begin{array}{l}400 \\
362 \\
311\end{array}$ & $\begin{array}{l}{ }^{1} \mathrm{~A}_{1} \mathrm{~g} \rightarrow{ }^{1} \mathrm{~B}_{1} \mathrm{~g} \\
{ }^{1} \mathrm{~A}_{1} \mathrm{~g} \rightarrow{ }^{1} \mathrm{Eg} \\
\text { C.T }\end{array}$ & $\mathrm{Sp}$ \\
\hline 8 & {$\left[\mathrm{Pt}(\mathrm{PPD})_{2} \mathrm{Cl}_{2}\right]$} & $\begin{array}{l}21459 \\
24875 \\
30769 \\
36363\end{array}$ & $\begin{array}{l}466 \\
402 \\
325 \\
275\end{array}$ & $\begin{array}{l}{ }^{1} \mathrm{~A}_{1} \mathrm{~g} \rightarrow{ }^{1} \mathrm{~A}_{2} \mathrm{~g} \\
{ }^{1} \mathrm{~A}_{1} \mathrm{~g} \rightarrow{ }^{1} \mathrm{~B}_{1} \mathrm{~g} \\
{ }^{1} \mathrm{~A}_{1} \mathrm{~g} \rightarrow{ }^{1} \mathrm{Eg} \\
\text { C.T } \mathrm{T}\end{array}$ & $\mathrm{Sp}$ \\
\hline 9 & {$\left[\mathrm{Pt}(\mathrm{PBD})_{2} \mathrm{Cl}_{2}\right]$} & $\begin{array}{l}21692 \\
25000 \\
32573 \\
36900\end{array}$ & $\begin{array}{l}461 \\
400 \\
307 \\
271\end{array}$ & $\begin{array}{l}{ }^{1} \mathrm{~A}_{1} \mathrm{~g} \rightarrow{ }^{1} \mathrm{~A}_{2} \mathrm{~g} \\
{ }^{1} \mathrm{~A}_{1} \mathrm{~g} \rightarrow{ }^{1} \mathrm{~B}_{1} \mathrm{~g} \\
{ }^{1} \mathrm{~A}_{1} \mathrm{~g} \rightarrow{ }^{1} \mathrm{Eg} \\
\text { C.T }\end{array}$ & $\mathrm{Sp}$ \\
\hline 10 & {$\left[\mathrm{Pt}(\mathrm{APOD})_{2} \mathrm{Cl}_{2}\right]$} & $\begin{array}{l}21739 \\
24937 \\
29850 \\
38022\end{array}$ & $\begin{array}{l}460 \\
401 \\
335 \\
263\end{array}$ & $\begin{array}{l}{ }^{1} \mathrm{~A}_{1} \mathrm{~g} \rightarrow{ }^{1} \mathrm{~A}_{2} \mathrm{~g} \\
{ }^{1} \mathrm{~A}_{1} \mathrm{~g} \rightarrow{ }^{1} \mathrm{~B}_{1} \mathrm{~g} \\
{ }^{1} \mathrm{~A}_{1} \mathrm{~g} \rightarrow{ }^{1} \mathrm{Eg} \\
\text { C.T }\end{array}$ & $\mathrm{Sp}$ \\
\hline 11 & {$\left[\mathrm{Pt}(\mathrm{DAPO})_{2} \mathrm{Cl}_{2}\right]$} & $\begin{array}{l}24390 \\
32509\end{array}$ & $\begin{array}{l}410 \\
307\end{array}$ & $\begin{array}{l}{ }^{1} \mathrm{~A}_{1} \mathrm{~g} \rightarrow{ }^{1} \mathrm{~B}_{1} \mathrm{~g} \\
{ }^{1} \mathrm{~A}_{1} \mathrm{~g} \rightarrow{ }^{1} \mathrm{Eg}\end{array}$ & $\mathrm{Sp}$ \\
\hline
\end{tabular}

Table 3. IR spectra for ligands and the prepared complexes.

\begin{tabular}{llllllll}
\hline Compound & $\boldsymbol{v}(\mathbf{O}-\mathbf{H})$ & $\mathbf{v}\left(\mathbf{N H}_{\mathbf{2}}\right)$ & & $\mathbf{v}(\mathbf{C}=\mathbf{N})$ & $\mathbf{v}(\mathbf{C}=\mathbf{C})$ & $\mathbf{v}(\mathbf{C}-\mathbf{O}-\mathbf{C})$ & $\mathbf{v}(\mathbf{M}-\mathbf{N})$ \\
\hline PPD & - & - & - & 1610 & 1544 & 1068 & - \\
PBD & - & - & - & 1613 & 1546 & 1066 & - \\
APOD & - & 3396 & 3269 & 1653 & 1568 & 1066 & - \\
DAPO & - & - & - & 1618 & 1500 & 1070 & - \\
1 & 3377 & - & - & 1602 & 1548 & 1070 & 423 \\
2 & 3357 & - & - & 1606 & 1541 & 1066 & 435 \\
3 & - & 3369 & 3278 & 1648 & 1564 & 1070 & 490,534 \\
4 & 3410 & - & - & 1607 & 1500 & 1070 & 525 \\
5 & - & - & - & 1606 & 1560 & 1090 & 432 \\
6 & - & 3492 & 3269 & 1645 & 1568 & 1066 & 428 \\
7 & - & - & - & 1610 & 1508 & 1082 & 420 \\
8 & - & - & - & 1603 & 1550 & 1082 & 441 \\
9 & - & - & - & 1608 & 1550 & 1091 & 436 \\
10 & - & 3402 & 3274 & 1643 & 1568 & 1071 & 416 \\
11 & - & - & - & 1606 & 1501 & 1076 & 503 \\
\hline
\end{tabular}

Table 4. ${ }^{1}$ HNMR data for prepared complexes.

\begin{tabular}{|c|c|c|c|}
\hline Ligand & Chemical shift & Complexes & Chemical shift \\
\hline \multirow[t]{2}{*}{ PPD } & $\begin{array}{l}\left.\text { 7.65(t, 6H, } \mathrm{C}_{6} \mathrm{H}_{5-}\right) \\
7.98\left(\mathrm{~d}, 4 \mathrm{H}, \mathrm{C}_{6} \mathrm{H}_{5-}\right)\end{array}$ & 1 & $\begin{array}{l}\left.\text { 7.62-7.94(m, } 8 \mathrm{H}, \mathrm{C}_{6} \mathrm{H}_{5-}\right) \\
8.122\left(\mathrm{~d}, 2 \mathrm{H}, \mathrm{C}_{6} \mathrm{H}_{5-}\right)\end{array}$ \\
\hline & & 8 & $\begin{array}{l}\left.\text { 7.63-7.93(m, } 8 \mathrm{H}, \mathrm{C}_{6} \mathrm{H}_{5-}\right) \\
\left.\text { 8.131(d, } 2 \mathrm{H}, \mathrm{C}_{6} \mathrm{H}_{5-}\right)\end{array}$ \\
\hline \multirow[t]{3}{*}{ PBD } & $\begin{array}{l}\left.\text { 7.38-7.75(m, } 10 \mathrm{H}, \mathrm{C}_{6} \mathrm{H}_{5-}\right) \\
8.08\left(\mathrm{~d}, 4 \mathrm{H}, \mathrm{C}_{6} \mathrm{H}_{5-}\right)\end{array}$ & 2 & $\begin{array}{l}\left.\text { 7.40-8.09(m, } 12 \mathrm{H}, \mathrm{C}_{6} \mathrm{H}_{5-}\right) \\
8.29\left(\mathrm{~d}, 2 \mathrm{H}, \mathrm{C}_{6} \mathrm{H}_{5-}\right)\end{array}$ \\
\hline & & 5 & $\begin{array}{l}\left.\text { 7.48-8.1(m, } 12 \mathrm{H}, \mathrm{C}_{6} \mathrm{H}_{5-}\right) \\
8.24\left(\mathrm{~d}, 2 \mathrm{H}, \mathrm{C}_{6} \mathrm{H}_{5-}\right)\end{array}$ \\
\hline & & 9 & $\begin{array}{l}\text { 7.5-8.1 }\left(\mathrm{m}, 12 \mathrm{H}, \mathrm{C}_{6} \mathrm{H}_{5-}\right) \\
8.24\left(\mathrm{~d}, 2 \mathrm{H}, \mathrm{C}_{6} \mathrm{H}_{5-}\right)\end{array}$ \\
\hline
\end{tabular}




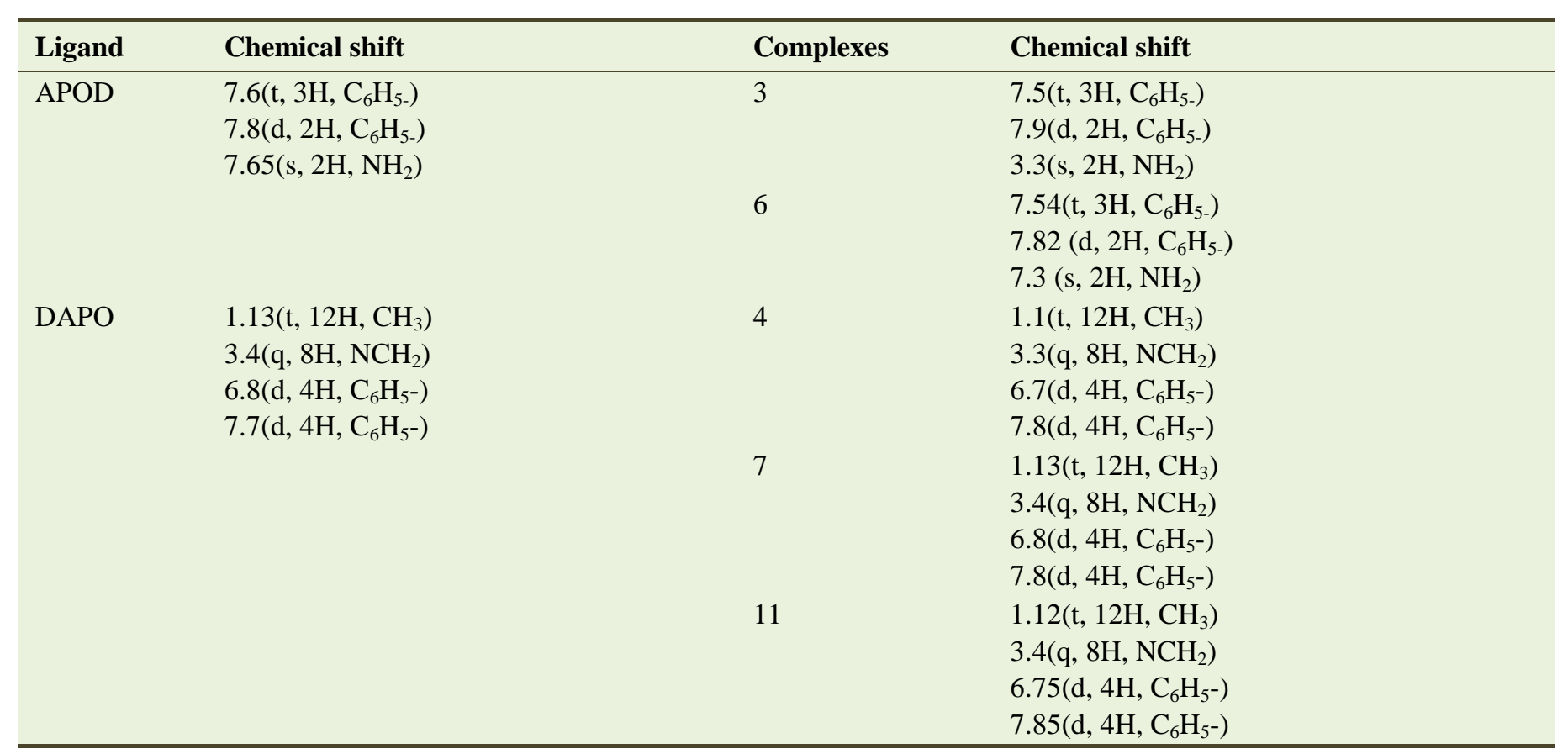

Table 5. ${ }^{13} \mathrm{CNMR}$ for prepared complexes.

\begin{tabular}{llll}
\hline Compound & $\boldsymbol{\delta C}$ (oxadiazole) $\mathbf{p p m}$ & $\boldsymbol{\delta} \mathbf{C}$ (phenyl) $\mathbf{p p m}$ & $\boldsymbol{\delta} \mathbf{C}$ (aliphatic) $\mathbf{p p m}$ \\
\hline PPD & 164 & $125-133$ & - \\
PBD & 164 & $125-140$ & - \\
APOD & $164-169$ & $125-129$ & - \\
DAPO & 164 & $115-149$ & $12-47$ \\
1 & 163 & $123-132$ & - \\
2 & 164 & $123-143$ & - \\
3 & 167 & $125-130$ & - \\
4 & 163 & $109-149$ & $12-46$ \\
5 & 163 & $143-123$ & - \\
6 & 167 & $122-131$ & - \\
7 & 163 & $107-149$ & - \\
8 & 164 & $126-133$ & - \\
9 & 165 & $126-141$ & - \\
10 & 165 & $125-132$ & $12-43$ \\
11 & 164 & $109-149$ & \\
\hline
\end{tabular}

Fig. 2: Various spectral analyses of metal(II) complexes.
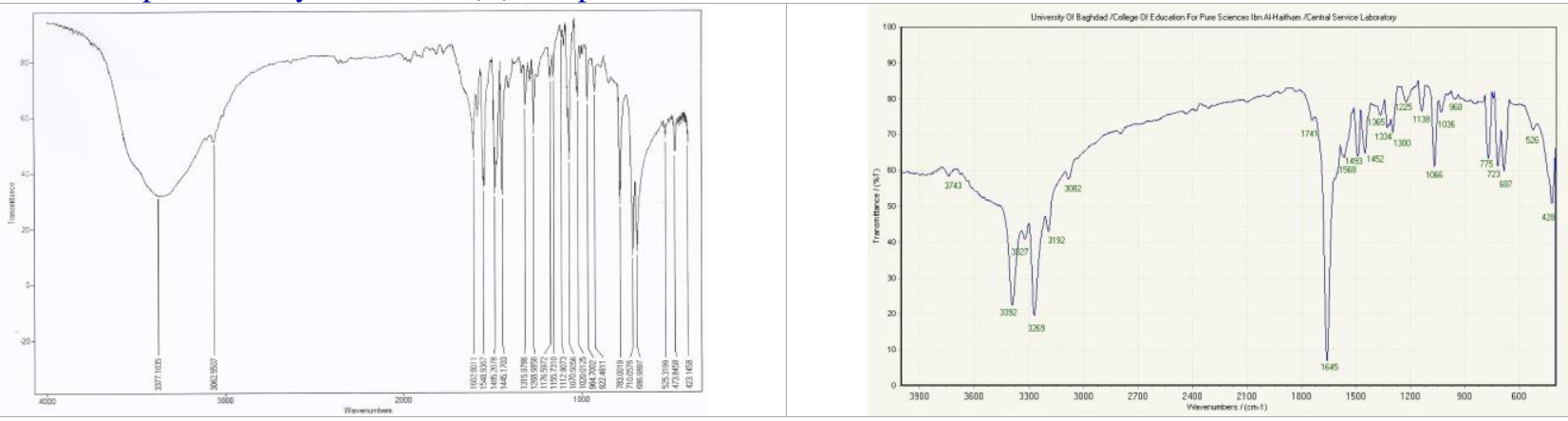


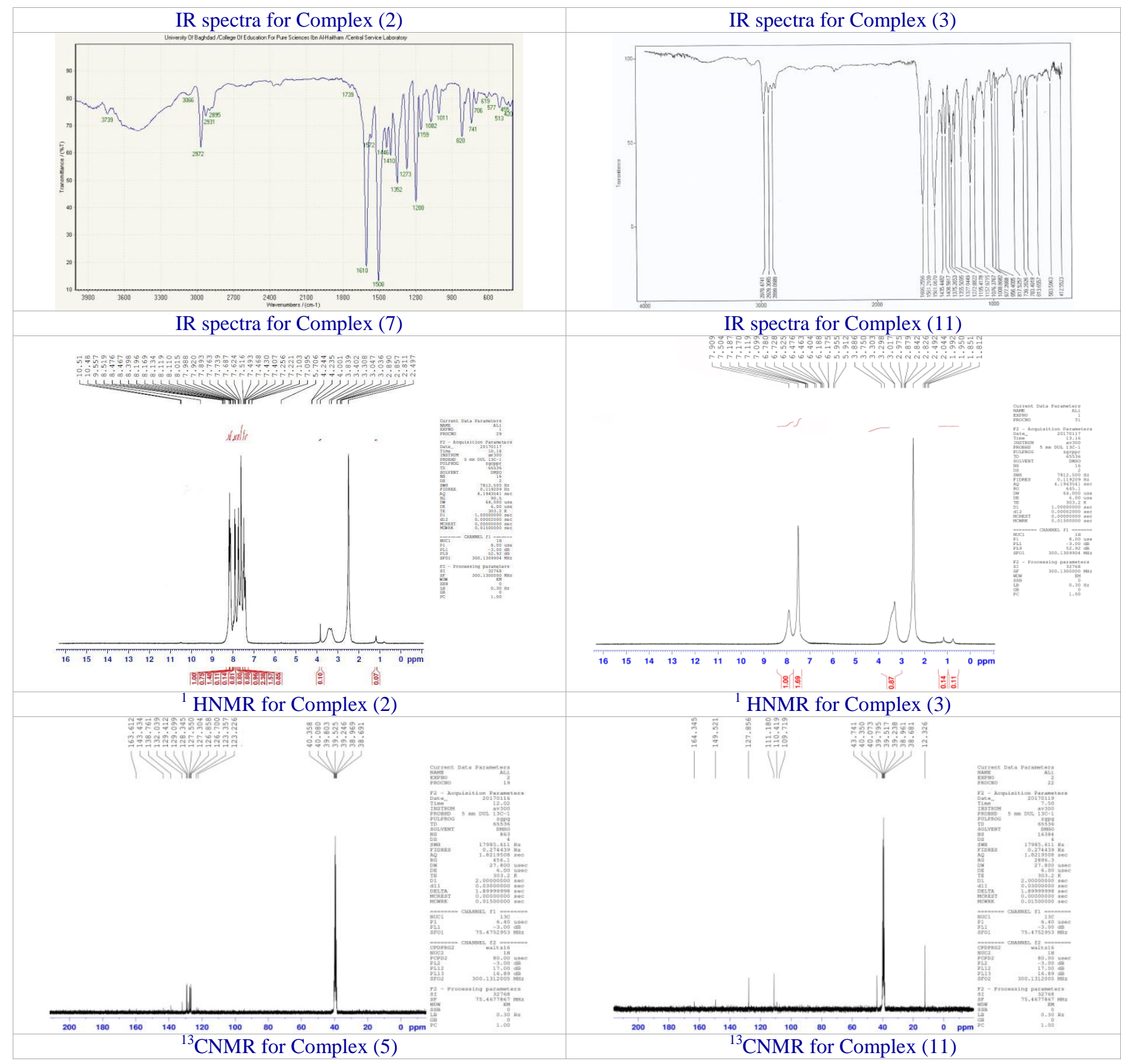

${ }^{13}$ CNMR data: ${ }^{13} \mathrm{CNMR}$ of the ligand recorded in $\mathrm{d}_{6-}$ (DMSO) solution. All ligand show signal at (164-169) ppm due to the carbon atom of the oxadiazole ring, and signal in rang (109-149) ppm due to the aromatic ring (Table 5). No change appeared in this signal in complexes formation. And this refers to uncoordinated aromatic carbon in the metal complexes (Abel et al., 1977).

\section{Conclusion}

The present work includes synthesis of complexes with
$\mathrm{Ni}(\mathrm{II}), \mathrm{Pd}(\mathrm{II})$ and $\mathrm{Pt}(\mathrm{II})$ with four 1,3,4-oxadiazole derivatives ligand. On the basis of IR, UV-Visible spectra, and magnetic susceptibility values, we concluded that the complexes (1-3) most probably to have octahedral geometries and complexes (4-11) probably have square planner geometries. According to the Molar conductivity data, it has been suggested that all synthesized complexes are non-electrolyte type. On the basis of the above discussions we propose the following structures for the metal (II) complexes as shown in Fig. 1. 


\section{Conflict of interest statement}

Authors declare that they have no conflict of interest.

\section{References}

Abel, E. W., Ahmed, A. K. S., Farrow, G. W., Orell, K. G., Sik, V., 1977. Nuclear magnetic resonance study of inversion at sulphur and selenium atoms in complexes of palladium(II) and platinum(II). Part 2. Complexes of methyl and phenyl trimethylsilylmethyl sulphide and selenide. J. Chem. Soc. Dalton Trans. 47, 47-52.

Aggarwal, R.C., Singh, N. K., 1975. Synthesis and characterization of picramates of $\mathrm{Cr}(\mathrm{III}), \mathrm{Mn}(\mathrm{II})$, $\mathrm{Fe}(\mathrm{II}), \mathrm{Co}(\mathrm{II}), \mathrm{Ni}(\mathrm{II}), \mathrm{Cu}(\mathrm{II})$ AND Zn(II). Indian J. Chem. 13, 931-934.

Al-Obaidi, K.H., Ali, B.F., Abu-El-Halawa, R., AboAmer, A., 2004. Synthesis of 1,3,4-mercaptooxadiazole mono- and dinuclear copper(I) and copper(II) complexes and their microbiological activity. Trans. Metal Chem. 29(7), 804-811.

Bala, S., Kamoboj, S., Kumar, A., 2010. Heterocyclic 1, 3, 4-oxadiazole compounds with diverse biological activities: A comprehensive review. J. Pharm. Res. 3(12), 2993-2997.

Blake, A.J., Gould, R.O., Halcrow, M.A., Schröder, M., 1993. Nickel thioether chemistry: synthesis, structures and electrochemistry of five-co-ordinate nickel(II) complexes of [9] $\mathrm{aneS}_{3}$. Crystal structures of $\quad\left[\mathrm{Ni}\left([9] \mathrm{aneS}_{3}\right)-(\mathrm{dppm})\right]\left[\mathrm{PF}_{6}\right]_{2^{\prime}}, \quad\left[\mathrm{Ni}\left([9] \mathrm{aneS}_{3}\right)\right.$ (dcpe) $\left[\mathrm{PF}_{6}\right]_{2} \cdot 1.25 \mathrm{MeCN}$ and $\left[\mathrm{Ni}\left([9] \mathrm{aneS}_{3}\right)\right.$ (tdpme) $\left[\mathrm{PF}_{6}\right]_{2}\left\{[9] \mathrm{aneS}_{3}=1,4,7-\right.$ Trithiacyclononane, $\mathrm{dppm}=\mathrm{Ph}_{2} \mathrm{PCH}_{2} \mathrm{PPh}_{2}$, depe $=\left(\mathrm{C}_{6} \mathrm{H}_{11}\right)_{2} \mathrm{PC}_{2} \mathrm{H}_{4} \mathrm{P}$ $\left(\mathrm{C}_{6} \mathrm{H}_{11}\right)_{2}$, tdpme $\left.=\mathrm{CH}_{3} \mathrm{C}\left(\mathrm{CH}_{2} \mathrm{PPh}_{2}\right)_{3}\right\}$. J. Chem. Soc. Dalton. Trans. 19, 2909-2920.

Cotton, F., Wikinson, G., 1999. Advanced Inorganic Chemistry. $6^{\text {th }}$ Edn., John Wiley and Sons, New York. pp.851-866.

Galal, S. A., Abdelsamie, A.S., Radriguez, L., Kerwin, S. M., El-Diwan, H. I., 2010. Synthesis and studying the antitumor activity of novel 5-(2methylbenzimidazol-5-yl)-1, 3, 4-oxadiazole-2 (3H)-thiones. Eur. J. Chem. 1(2), 67-72.
Garces, F. O., King, K. A., Watts, R. J., 1988. Synthesis, structure, electrochemistry, and photophysics of methyl-substituted phenylpyridine ortho-metalated iridium(III) complexes. Inorg. Chem. 27(10), 34643471.

Gudasi, K., Patil, M., Vadavi, R., Shenoy, R., Patil, S., 2007. Transition metal complexes with a new tridentate ligand, 5-[6-(5-mercapto-1, 3, 4oxadiazol-2-yl) pyridin-2-yl]-1, 3, 4-oxadiazole-2thiol. J. Serb. Chem. Soc. 72 (4), 357-366.

Kavitha, S., Gnanavel, S., Kannan, K., 2014. Biological aspects of 1,3,4-oxadiazole derivatives. Asian J. Pharmaceut. Clin. Res. 7(4), 11-20.

Levason, W., McAuliffe, C.A., Murray, S.G., 1976. Palladium(II) complexes of o-phenylenebis (diphenylstibine) and the tendency of group VB ligands to promote penta coordination. Inorg. Nucl. Chem. Lett. 12(11), 849-853.

Park, Y.C., Cho, Y.J., 1989. Bull. Kor. Chem. Soc. 10, 220.

Patricia, G.S., Javier, G.T., Miguel, A.M., Francisco, J.A., Teofilo, R., 2002. Evidence of desulfurization in the oxidative cyclization of thiosemicarbazones. Conversion to 1,3,4-oxadiazole derivatives. Inorg. Chem. 41(6), 1345-1347.

Qadir, A. M., Abdullah, A. I., Al-Jibori, S. A., Al-Allaf, T. A. K., 2004. Palladium(II) and platinum(II) complexes with mixed ligands of tertiary monophosphines and 5-phenyl-1,3,4-oxadiazole-2thione or 4,5-diphenyl-1,2,4-triazole-3-thione. Asian J. Chem. 16, 1181-1188.

Somani, R. R., Shirodkar, P. Y., 2009. Oxadiazole: A biologically active heterocycles. Der Pharma Chemica. 1(1), 130-140.

Subha, C., Selvaraj, A., 2014. Synthesis and characterization of $\mathrm{Co}$ (Ii) and $\mathrm{Ni}$ (Ii) complexes of 2, 5-substituted 1, 3, 4-oxadiazole derivatives. Res. J. Chem. Sci. 4(12), 34-38.

Tomi, I. H. R., 2012. Synthesis, characterization and comparative study of mesomorphic properties of some new compounds containing both 1, 2, 4-and 1, 3, 4-oxadiazole moieties linked in the same molecule. J. Saudi Chem. Soc. 16(2), 153-159.

\section{How to cite this article:}

Mohamad, H. A., Al-Kattan, W. T., Najem, A. N., 2017. Synthesis and characterization platinum (II), palladium (II) and nickel (II) complexes with some heterocyclic ligand of 1,3,4-oxadazole derivative. Int. J. Curr. Res. Biosci. Plant Biol. 4(7), 67-74. doi: https://doi.org/10.20546/ijcrbp.2017.407.008 